Institute of Child Neuropsychiatry, University of Pisa, Via dei Giacinti 2, 56018 Calambrone, Pisa, Italy).

COMMENT. Vomiting as an ictal phenomenon is controversial and difficult to distinguish from migraine. In these patients with previous evidence of occipital epileptiform EEG discharges, visual symptoms followed by automatisms and vomiting appeared more likely to result from temporal lobe ictal involvement than a migraine secondary to an occipital seizure. The lateralization of the ictal discharge to the right hemisphere has previously been reported in 13 children with the diagnosis of ictus emeticus. (Kramer RE et al, 1988). Cyclical vomiting as a form of epilepsy in 33 children was described in 1955. (Millichap JG, Lombroso CT, Lennox WG). See Progress in Pediatric Neurology, Chicago, PNB Publishers, 1991, for further reports of ictus emeticus.

\title{
VALPROATE-INDUCED HEPATIC FAILURE WITH COX DEFICIT
}

A fatal hepatic failure in a 3 year-old girl with myoclonic epilepsy after 3 months of treatment with valproate (VPA) is reported from Hopital d'Enfants, Marseilles, and Hopital des Enfants-Malades, Paris, France. Elevated plasma lactate and lactate/pyruvate molar ratios in plasma suggested a defect in oxidative phosphorylation and prompted investigation of respiratory chain activity. Circulating lymphocytes revealed a cytochrome c oxidase (COX) deficiency, later confirmed by post-mortem analysis in liver and cultured skin fibroblasts. Skeletal muscle analysis was normal. (Chabrol B et al. Valproateinduced hepatic failure in a case of cytochrome $c$ oxidase deficiency. Eur I Pediatr 1994;153:133-135). (Respond: Dr B Chabrol, Service de Neuropediatrie, Hopital d'Enfants, CHU de la Timone, F-13385 Marseille Cedex 5, France).

COMMENT. Lactate/pyruvate plasma levels are recommended in children with possible mitochondrial disorders and epilepsy when VPA treatment is employed. Valproate associated hepatotoxicity is discussed in previous issues of Ped Neur Briefs Jan, May, and Aug 1993, and June 1987.

\section{BEHAVIOR DISORDERS}

\section{EARLY SIGNS OF AUTISM}

A blinded comparison of parental and clinical observations of the behavior of 26 autistic children ( 23 boys and 3 girls) younger than age 48 months is reported from the Vanderbilt University School of Medicine, Nashville, TN, and The Children's Mercy Hospital, Kansas City. Five most prevalent behavioral characteristics both reported by parents and observed by clinicians were as follows: 1) abnormal social play (eg. nonparticipation in peekaboo and itsy bitsy spider games); 2) lack of awareness of others (eg. noninteraction); 3) impaired imitation (eg. wave goodbye, patty-cake); 4) deficient nonverbal communication (eg. absent social smile or eye contact); and 5) absent imaginative play (eg. pretend games). Autistic behaviors rarely endorsed by parents and clinicians included: abnormal comfort seeking, abnormal speech, distress over change, and insistence on sameness and routines. Parents were more likely than clinicians to report absence of imaginative play and presence of stereotyped movements. (Stone WL, Hoffman EL et al. Early recognition of autism. Parental reports vs clinical observation. 
Arch Pediatr Adolesc Med Feb 1994;148:174-179). (Reprints: Dr Stone, Child Development Center, Vanderbilt University Medical Center S, Room 426, 2100 Pierce Ave, Nashville, TN 37232).

COMMENT. Improved awareness of early signs of autism should help physicians recognize and refer patients for specialized intervention. Parents are better judges of a child's imaginative play and peer friendships, whereas physicians may be more objective about a child's social awareness, interactive play, imitation skills, and nonverbal communication.

Decreased plasma concentrations of the C4B complement protein are reported in a group of 42 autistic subjects examined at the Center for Persons with Disabilities and Department of Biology, Utah State University, Logan, UT. (Warren RP et al. Arch Pediatr Adolesc Med Feb 1994; 148:180-183).

\section{ABNORMAL EEG IN AUTISM: VALPROATE RESPONSE}

Three children, ages 3, 4, and 5 years, with autism and epileptiform EEG discharges showed clinical improvement with valproic acid therapy at Mercy Hospital and Medical Center, Chicago, IL. None had a history of seizures. Within one month of VPA $125 \mathrm{mg}$ tid treatment, language and social skills improved and the DSM-III-R criteria for autism no longer applied. Improvement had been maintained at follow-up 7 to 11 months later. (Plioplys AV. Autism: electroencephalogram abnormalities and clinical improvement with valproic acid. Arch Pediatr Adolesc Med Feb 1994;148:220-222). (Reprints: Dr Plioplys, Division of Neurology, Mercy Hospital and Medical Center, Stevenson Expressway at King Drive, Chicago, IL 60616).

COMMENT. The author stresses the importance of sleep EEGs to uncover epileptiform discharges in young autistic patients without history of clinical seizures. Further trials of antiepileptic drugs in autistic children seem justified.

\section{POSTERIOR FOSSA ABNORMALITIES IN INFANTILE AUTISM}

Previously published cerebellar vermis measures of 78 autistic patients from 4 separate MRI studies have been reanalysed at the Neurosciences Department, School of Medicine, University of California at San Diego, La Jolla, CA. Abnormalities were in 2 groups: vermal hypoplasia in $80-90 \%$ and vermal hyperplasia in $8-16 \%$ patients. These subgroups also differed significantly from normal controls. Failure to recognize these variations in vermal structure among patients may have lead to disparate reports of cerebellar maldevelopment in infantile autism. (Courchesne E et al. The brain in infantile autism: Posterior fossa structures are abnormal. Neurology Feb 1994;44:214-223). (Reprints: Dr Eric Courchesne, Neuropsychology Research Laboratory, Children's Hospital, 3020 Children's Way, San Diego, CA 92123).

COMMENT. Cerebellar pathology and hypoplasia have been reported in Rett and Down syndromes as well as autism. Attentional asynergia and dysfunction following cerebellar damage are linked to impaired social communication skills. Cerebellar mutism and personality changes have followed surgical removal of medulloblastoma.(Ped Neur Briefs Feb 